\title{
Conformations, Protonation Sites, and Metal Complexation of Benzohidroxamic Acid. A Theoretical and Experimental Study
}

Begoña García ${ }^{\S *}$, Saturnino Ibeas ${ }^{\S}$, José M. Leal ${ }^{\S}$, Fernando Secco ${ }^{*}$, Marcella Venturini" ${ }^{\#}$ Maria L. Senent $\$$, Alfonso Niño* and Camelia Muñoz.*

\footnotetext{
contents:

Tab1e s1-s27

Figure $\mathrm{S} 1$

Figure s2

Figure s3
} 
Table s1. Z-anion Ia calculated with RHF/cc-pVDZ (Er=0.0 Kcal/mo1)

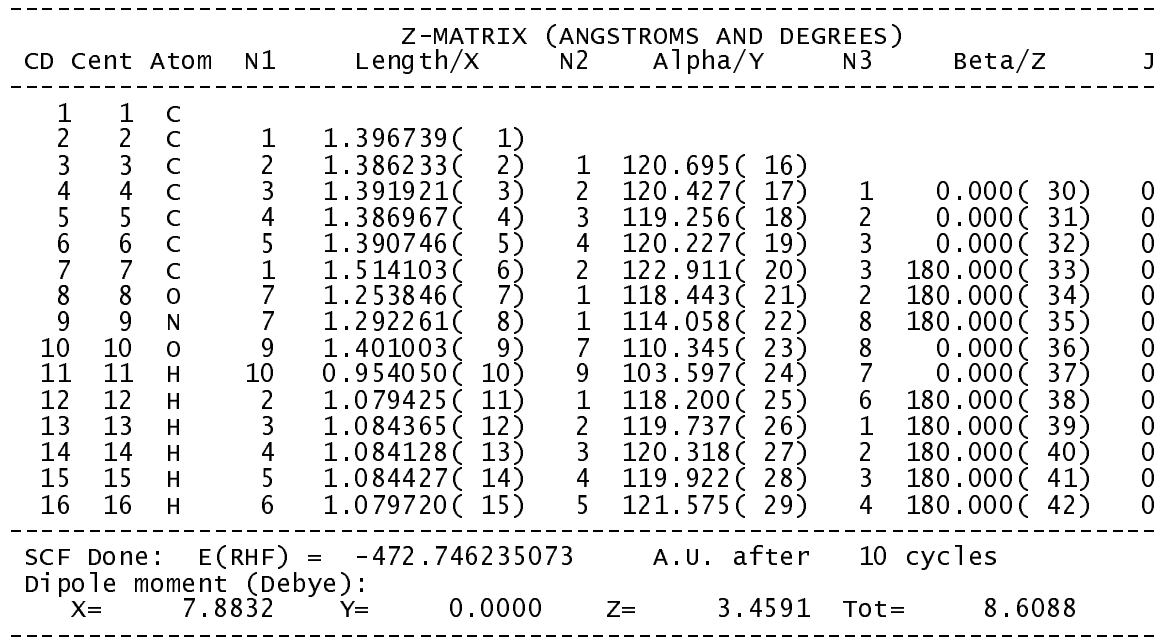

Table s2. Z-anion II calculated with RHF/cc-pVDZ (Er=25.6 kcal/mol)

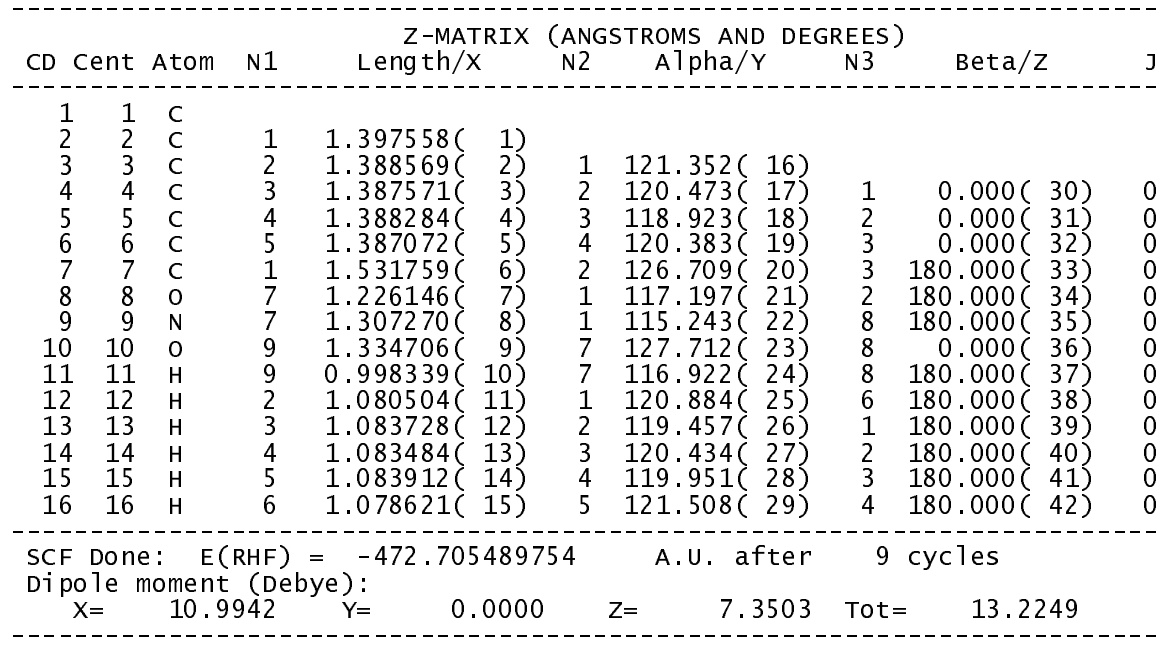

Table s3. Z-anion Ib calculated with RHF/cc-pVDZ (Er=20.0 Kcal/mol)

\begin{tabular}{|c|c|c|c|c|c|c|c|c|c|c|c|}
\hline$C D$ & Cent & Atom & $\mathrm{N} 1$ & \multicolumn{2}{|c|}{$\begin{array}{l}\text { Z-MATRIX } \\
\text { Leng th } / X\end{array}$} & \multicolumn{2}{|c|}{$\begin{array}{l}\text { (ANGSTROMS AND } \\
\text { N2 A1pha } / Y\end{array}$} & \multicolumn{2}{|c|}{$\begin{array}{c}\text { DEGREES) } \\
\text { N3 }\end{array}$} & Beta/z & \\
\hline $\begin{array}{r}1 \\
2 \\
3 \\
4 \\
5 \\
6 \\
7 \\
8 \\
10 \\
11 \\
12\end{array}$ & $\begin{array}{r}1 \\
2 \\
3 \\
4 \\
5 \\
6 \\
7 \\
8 \\
9 \\
10 \\
11 \\
12 \\
13 \\
14 \\
15 \\
16\end{array}$ & $\begin{array}{l}\mathrm{C} \\
\mathrm{C} \\
\mathrm{C} \\
\mathrm{C} \\
\mathrm{C} \\
\mathrm{C} \\
\mathrm{C} \\
\mathrm{O} \\
\mathrm{N} \\
\mathrm{O} \\
\mathrm{H} \\
\mathrm{H} \\
\mathrm{H} \\
\mathrm{H} \\
\mathrm{H} \\
\mathrm{H}\end{array}$ & $\begin{array}{l}1 \\
2 \\
3 \\
4 \\
5 \\
1 \\
7 \\
7 \\
9 \\
8 \\
2 \\
3 \\
4 \\
5 \\
6\end{array}$ & $\begin{array}{l}1.406140 \\
1.380255 \\
1.395787 \\
1.385270 \\
1.390628 \\
1.459028 \\
1.363269 \\
1.275070 \\
1.305016 \\
0.969674 \\
1.079636 \\
1.084533 \\
1.083421 \\
1.084661 \\
1.079797\end{array}$ & $\begin{array}{r}\text { 1) } \\
2) \\
3) \\
4) \\
5) \\
6) \\
7) \\
8) \\
9) \\
10) \\
11) \\
12) \\
13) \\
14) \\
15)\end{array}$ & $\begin{array}{l}1 \\
2 \\
3 \\
4 \\
2 \\
1 \\
1 \\
7 \\
7 \\
1 \\
2 \\
3 \\
4 \\
5\end{array}$ & 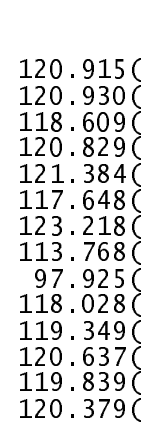 & $\begin{array}{l}16) \\
17) \\
18) \\
19) \\
20) \\
21) \\
22) \\
23) \\
24) \\
25) \\
26) \\
27) \\
28) \\
29)\end{array}$ & $\begin{array}{l}1 \\
2 \\
3 \\
3 \\
2 \\
8 \\
8 \\
9 \\
6 \\
1 \\
2 \\
3 \\
4\end{array}$ & 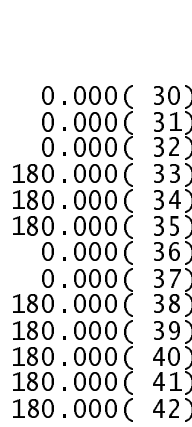 & \\
\hline & & & & $\mathrm{Y}=$ & & & $2-$ & & & $=9$. & \\
\hline
\end{tabular}


Table S4. E-anion Ia calculated with RHF/cc-pVDZ (Er=16.3 Kcal/mol)

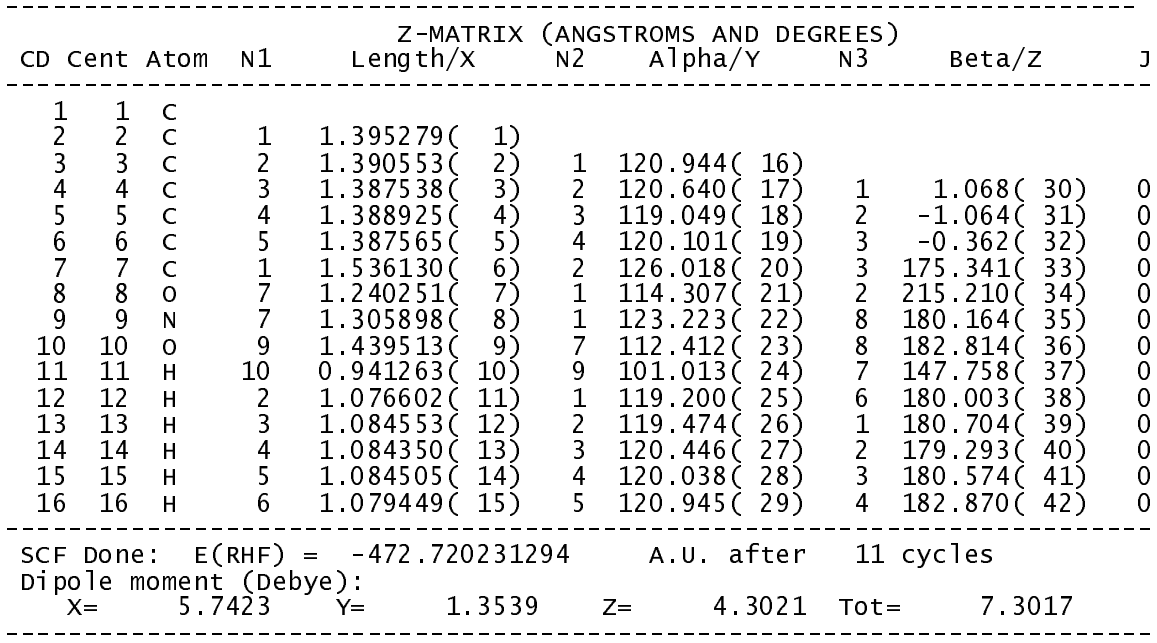

Table S5. E-anion Ib calculated with RHF/cc-pVDZ (Er=29.9 $\mathrm{kcal} / \mathrm{mol})$

\begin{tabular}{|c|c|c|c|c|c|c|c|c|c|c|}
\hline$C D$ & ent & Atom & N1 & $\begin{array}{r}\text { Z-MA } \\
\text { Length/ }\end{array}$ & IX & $\begin{array}{l}\text { (ANG } \\
\text { N2 }\end{array}$ & $\begin{array}{c}\text { TROMS AND DI } \\
\text { Alpha/Y }\end{array}$ & $\begin{array}{l}\text { ES) } \\
3\end{array}$ & Beta/Z & \\
\hline $\begin{array}{r}1 \\
2 \\
3 \\
4 \\
5 \\
6 \\
7 \\
8 \\
9 \\
10 \\
11 \\
12 \\
13 \\
14 \\
15 \\
16\end{array}$ & $\begin{array}{r}1 \\
2 \\
3 \\
4 \\
5 \\
6 \\
7 \\
8 \\
9 \\
10 \\
11 \\
12 \\
13 \\
14 \\
15 \\
16\end{array}$ & $\begin{array}{l}\mathrm{C} \\
\mathrm{C} \\
\mathrm{C} \\
\mathrm{C} \\
\mathrm{C} \\
\mathrm{C} \\
\mathrm{C} \\
\mathrm{O} \\
\mathrm{N} \\
\mathrm{O} \\
\mathrm{H} \\
\mathrm{H} \\
\mathrm{H} \\
\mathrm{H} \\
\mathrm{H} \\
\mathrm{H} \\
\mathrm{H}\end{array}$ & $\begin{array}{l}1 \\
2 \\
3 \\
4 \\
5 \\
1 \\
7 \\
7 \\
9 \\
8 \\
2 \\
3 \\
4 \\
5 \\
6\end{array}$ & $\begin{array}{l}1.405792 \\
1.386330 \\
1.390768 \\
1.388547 \\
1.386996 \\
1.463690 \\
1.397273 \\
1.273941 \\
1.287227 \\
0.946898 \\
1.077083 \\
1.084771 \\
1.084025 \\
1.084866 \\
1.079174\end{array}$ & $\begin{array}{l}1) \\
2) \\
3) \\
4) \\
5) \\
65 \\
75 \\
8) \\
99 \\
10) \\
11) \\
12) \\
13) \\
145 \\
15)\end{array}$ & $\begin{array}{l}1 \\
2 \\
3 \\
4 \\
2 \\
1 \\
1 \\
7 \\
7 \\
1 \\
2 \\
3 \\
4 \\
5\end{array}$ & 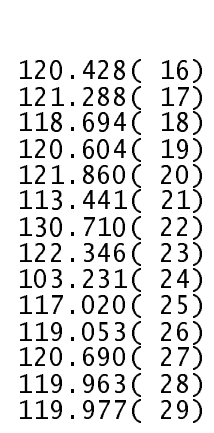 & $\begin{array}{l}1 \\
2 \\
3 \\
3 \\
2 \\
8 \\
8 \\
9 \\
6 \\
1 \\
2 \\
3 \\
4\end{array}$ & $\begin{array}{r}0.000(30) \\
0.000(31) \\
0.000(32) \\
180.000(33) \\
180.000(34) \\
180.000(35) \\
180.000(36) \\
0.000(37) \\
180.000(38) \\
180.000(39) \\
180.000(40) \\
180.000(41) \\
180.000(42)\end{array}$ & \\
\hline & & & & $Y=-472$. & & & $\mathrm{Z}=\begin{array}{r}\mathrm{A} \cdot \mathrm{U} \cdot \mathrm{aft} \\
-1.38\end{array}$ & $\begin{array}{l}10 \\
\text { Tot }=\end{array}$ & $=\begin{array}{r}\text { cycles } \\
7.2\end{array}$ & \\
\hline
\end{tabular}

Table s6. E-anion II calculated with RHF/cc-pVDZ (Er=13.8 kcal/mol)

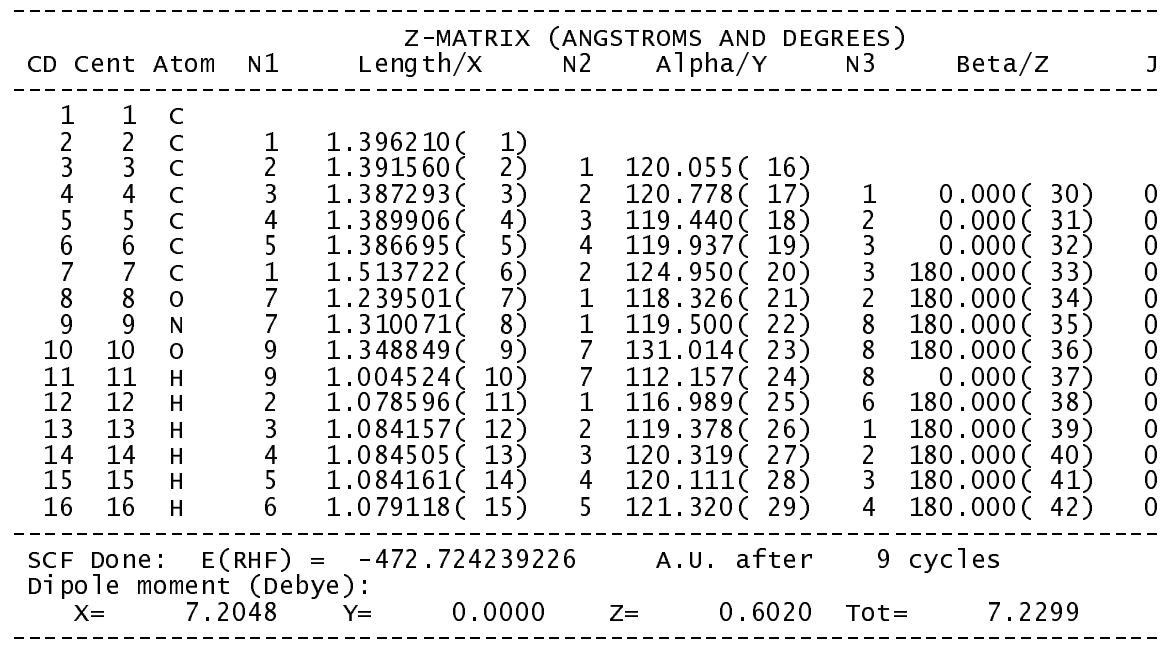


Table s7. Z-AMIDE calculated with RHF/cc-pVDZ (Er=0 kcal/mol)

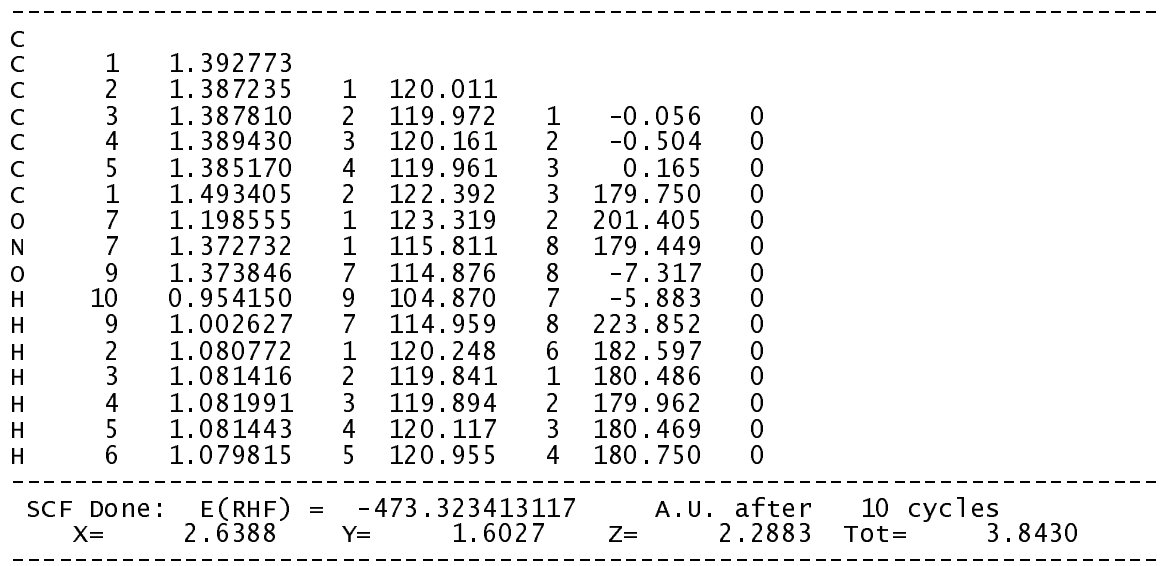

Table s8. E-AMIDE calculated with RHF/cc-pVDZ (Er=3.7 $\mathrm{kcal} / \mathrm{mol}$ )

\begin{tabular}{|c|c|c|c|c|c|c|}
\hline 1 & $\begin{array}{l}1 \\
2 \\
3 \\
4 \\
5 \\
1 \\
7 \\
7 \\
9 \\
0 \\
9 \\
2 \\
3 \\
4 \\
5 \\
6\end{array}$ & $\begin{array}{l}1.393728 \\
1.387342 \\
1.387746 \\
1.388878 \\
1.385060 \\
1.497619 \\
1.196032 \\
1.378941 \\
1.367567 \\
0.949874 \\
1.002748 \\
1.077919 \\
1.081585 \\
1.082204 \\
1.081539 \\
1.079713\end{array}$ & $\begin{array}{l}1 \\
2 \\
3 \\
4 \\
2 \\
1 \\
1 \\
7 \\
9 \\
7 \\
1 \\
2 \\
3 \\
4 \\
5\end{array}$ & $\begin{array}{l}119.952 \\
120.209 \\
120.072 \\
119.824 \\
123.804 \\
122.049 \\
119.262 \\
121.056 \\
108.310 \\
113.361 \\
120.304 \\
119.668 \\
119.936 \\
120.225 \\
120.718\end{array}$ & $\begin{array}{l}1 \\
2 \\
3 \\
3 \\
2 \\
8 \\
8 \\
7 \\
8 \\
6 \\
1 \\
2 \\
3 \\
4\end{array}$ & $\begin{array}{r}0.578 \\
-0.757 \\
-0.167 \\
176.592 \\
206.319 \\
177.579 \\
206.465 \\
66.297 \\
-13.936 \\
180.903 \\
180.619 \\
179.576 \\
180.457 \\
181.570\end{array}$ \\
\hline
\end{tabular}

Table s9. Z-IMIDE calculated with RHF/cc-pVDZ (Er=3.4 kcal/mol)

\begin{tabular}{|c|c|c|c|c|c|c|c|c|c|}
\hline $\begin{array}{l}\mathrm{C} \\
\mathrm{C} \\
\mathrm{C} \\
\mathrm{C} \\
\mathrm{C} \\
\mathrm{C} \\
\mathrm{O} \\
\mathrm{N} \\
\mathrm{O} \\
\mathrm{H} \\
\mathrm{H} \\
\mathrm{H} \\
\mathrm{H} \\
\mathrm{H} \\
\mathrm{H} \\
\mathrm{H}\end{array}$ & $\begin{array}{r}1 \\
2 \\
3 \\
4 \\
5 \\
1 \\
7 \\
7 \\
9 \\
10 \\
8 \\
2 \\
3 \\
4 \\
5 \\
6\end{array}$ & $\begin{array}{l}1.395212 \\
1.383156 \\
1.391133 \\
1.385270 \\
1.389277 \\
1.482350 \\
1.328621 \\
1.261562 \\
1.385548 \\
0.943814 \\
0.950567 \\
1.079221 \\
1.081712 \\
1.082057 \\
1.081675 \\
1.078961\end{array}$ & $\begin{array}{l}1 \\
2 \\
3 \\
4 \\
2 \\
1 \\
1 \\
7 \\
9 \\
7 \\
1 \\
2 \\
3 \\
4 \\
5\end{array}$ & $\begin{array}{l}120.102 \\
120.192 \\
119.860 \\
120.129 \\
120.439 \\
115.321 \\
120.726 \\
110.227 \\
104.365 \\
108.216 \\
119.338 \\
119.734 \\
120.033 \\
120.187 \\
120.136\end{array}$ & $\begin{array}{l}1 \\
2 \\
3 \\
3 \\
2 \\
8 \\
8 \\
7 \\
9 \\
6 \\
1 \\
2 \\
3 \\
4\end{array}$ & $\begin{array}{r}-0.004 \\
0.014 \\
-0.008 \\
179.995 \\
179.928 \\
180.014 \\
0.007 \\
180.014 \\
-0.058 \\
179.993 \\
180.007 \\
180.015 \\
179.995 \\
180.005\end{array}$ & $\begin{array}{l}0 \\
0 \\
0 \\
0 \\
0 \\
0 \\
0 \\
0 \\
0 \\
0 \\
0 \\
0 \\
0 \\
0\end{array}$ & & \\
\hline & & $\begin{array}{c}\text { E(RHF) } \\
\text { oment (Deb } \\
-0.1788\end{array}$ & $\mathrm{Y}=$ & $\begin{array}{r}473.31806 \\
0.00\end{array}$ & 987 & A. & $\begin{array}{l}\text { after } \\
0.1720\end{array}$ & Tot $=$ & $\begin{array}{l}\text { es } \\
0.2481\end{array}$ \\
\hline
\end{tabular}


Table S10. E-IMIDE calculated with RHF/cc-pVDZ (Er=10.1 kcal/mo1)

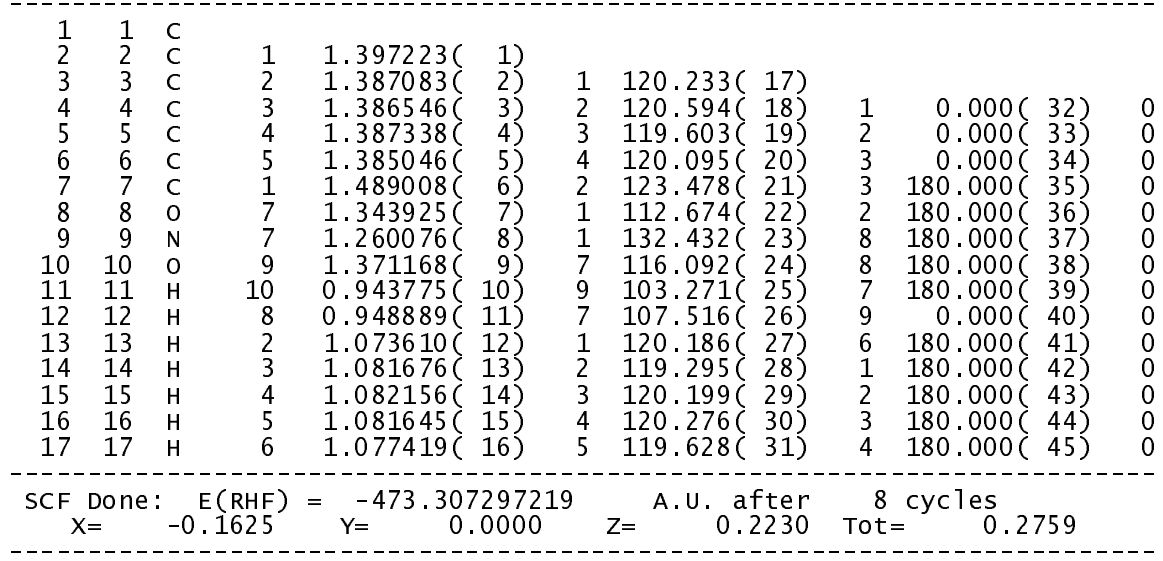

Table s11. Z-AMIDE calculated with RHF/cc-pVDZ (not absolute minime)

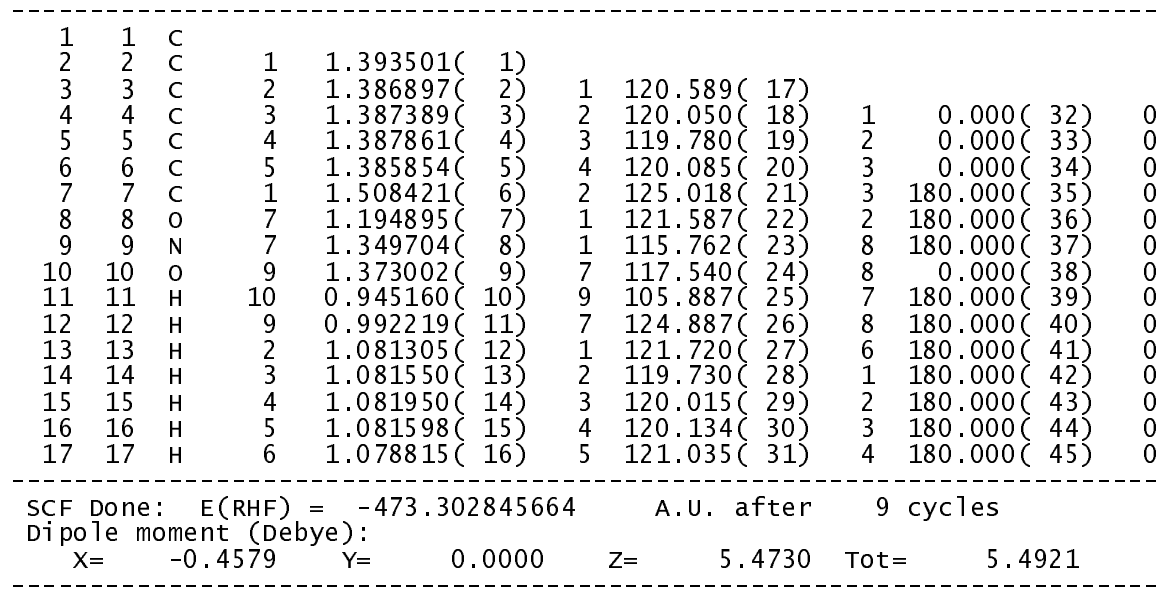

Table S12. E-AMIDE calculated with RHF/cc-pVDZ (not absolute minime)

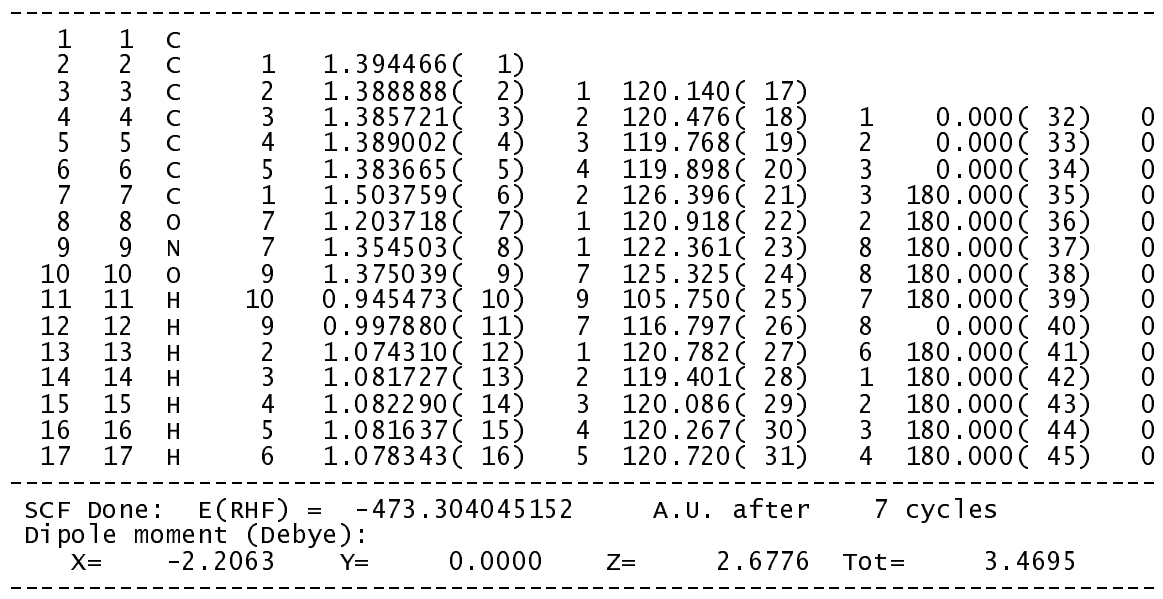


Table s13. Z-IMIDE calculated with RHF/cc-pVDZ (not absolute minime)

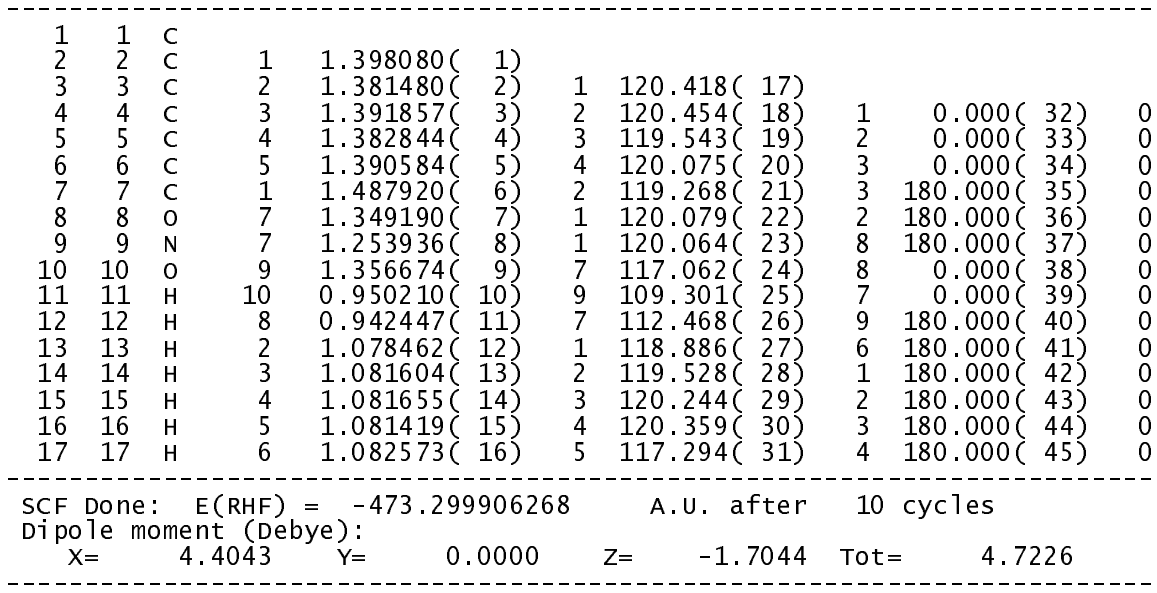

Table s14. E-IMIDE calculated with RHF/cc-pVDZ (not absolute minime)

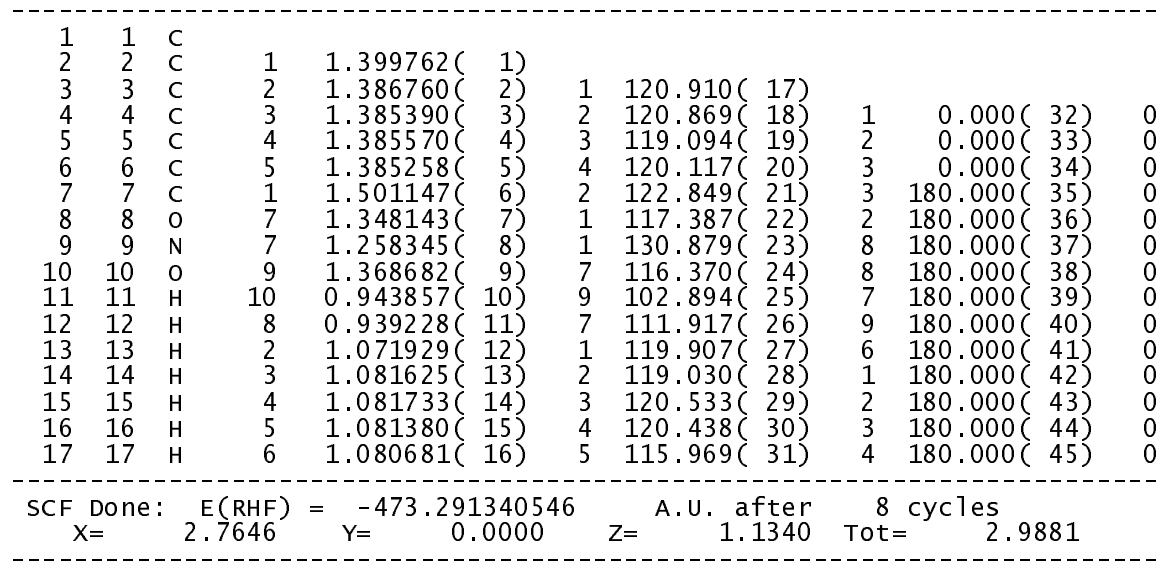

Table s15. E-IMIDE calculated with RHF/cc-pVDZ (not absolute minime)

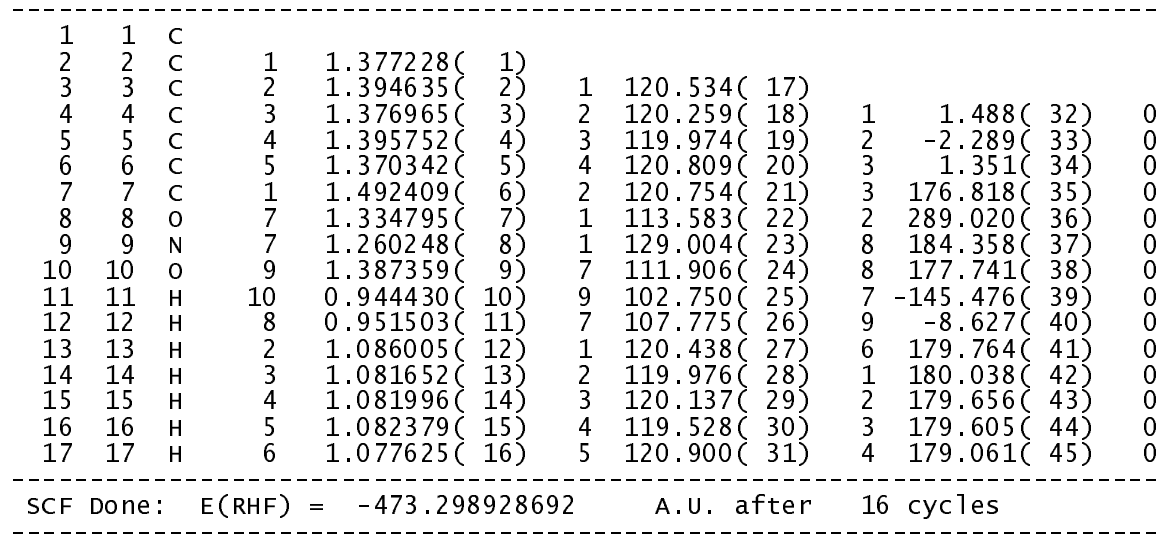


Table S16. E-IMIDE calculated with RHF/cc-pVDZ (not absolute minime)

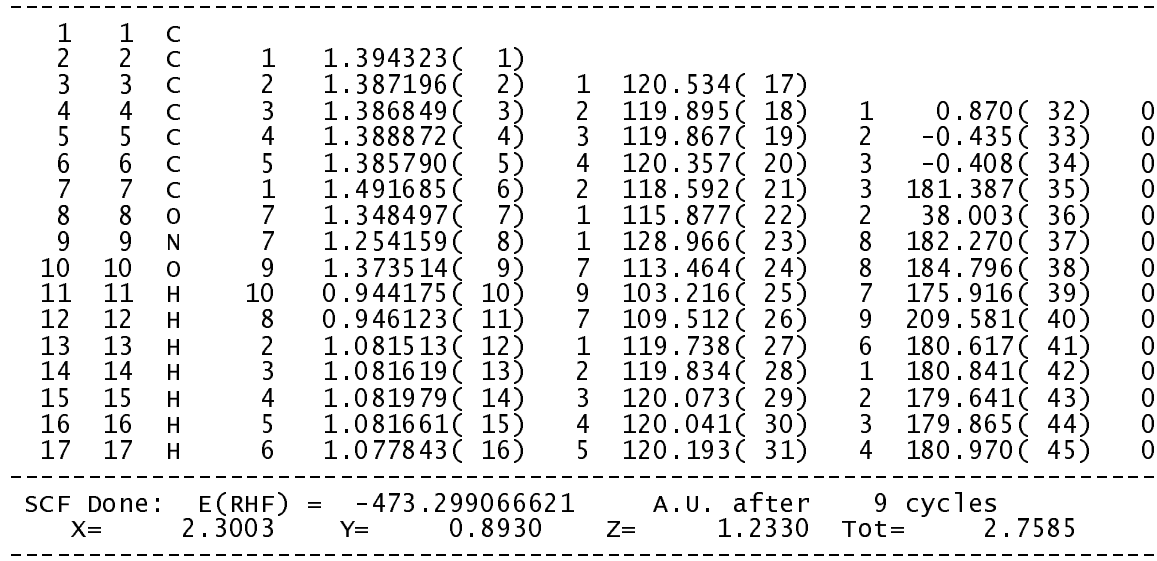

Table s17. Z-AMIDE calculated with DFT/AUG-cc-pVDZ

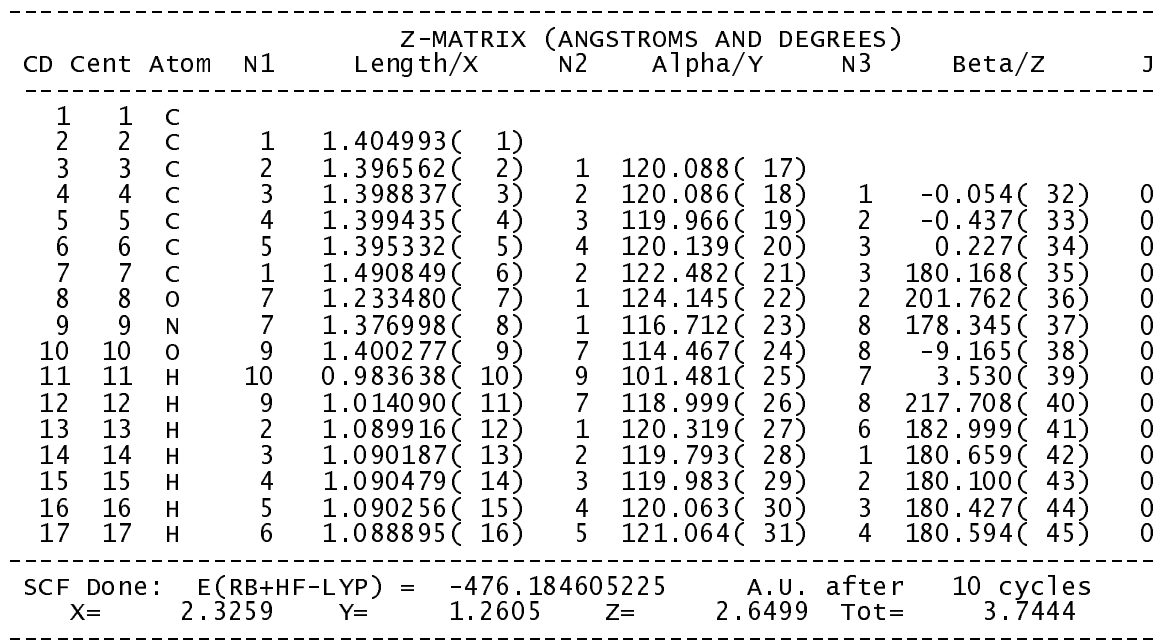

Table s18. Z-IMIDE calculated with DFT/AUG-cc-pVDZ

\begin{tabular}{|c|c|c|c|c|c|c|c|}
\hline$C D$ & Cent & t Atom N1 & & $\begin{array}{r}Z-M A T \\
\text { Length } / X\end{array}$ & RIX & $\begin{array}{ccc}\text { (ANGSTROMS AND } & \text { DEGREES) } \\
\mathrm{N} 2 & \mathrm{~A} 7 \mathrm{pha} / \mathrm{Y} & \mathrm{N} 3\end{array}$ & Beta $/ Z$ \\
\hline $\begin{array}{l}6 \\
6 \\
6 \\
6 \\
6 \\
6 \\
6 \\
8 \\
7 \\
8 \\
1 \\
1 \\
1 \\
1 \\
1 \\
1 \\
1\end{array}$ & $\begin{array}{r}1 \\
2 \\
3 \\
4 \\
5 \\
1 \\
7 \\
7 \\
9 \\
10 \\
8 \\
2 \\
3 \\
4 \\
5 \\
6\end{array}$ & $\begin{array}{l}1.406934 \\
1.393430 \\
1.401108 \\
1.396930 \\
1.397727 \\
1.476736 \\
1.352467 \\
1.290878 \\
1.422331 \\
0.963980 \\
0.971779 \\
1.088244 \\
1.090405 \\
1.090526 \\
1.090375 \\
1.087903\end{array}$ & $\begin{array}{l}1 \\
2 \\
3 \\
4 \\
2 \\
1 \\
1 \\
7 \\
9 \\
7 \\
1 \\
2 \\
3 \\
4 \\
5\end{array}$ & $\begin{array}{l}120.089 \\
120.351 \\
119.718 \\
120.241 \\
120.593 \\
115.777 \\
120.721 \\
109.029 \\
103.051 \\
107.043 \\
119.384 \\
119.637 \\
120.113 \\
120.136 \\
120.194\end{array}$ & $\begin{array}{l}1 \\
2 \\
3 \\
3 \\
2 \\
8 \\
8 \\
7 \\
9 \\
6 \\
1 \\
2 \\
3 \\
4\end{array}$ & $\begin{array}{r}0.000 \\
0.000 \\
0.000 \\
180.000 \\
180.000 \\
180.000 \\
0.000 \\
180.000 \\
0.000 \\
180.000 \\
180.000 \\
180.000 \\
180.000 \\
180.000\end{array}$ & \\
\hline & $F=$ & $\begin{array}{l}\mathrm{E}(\mathrm{RB}+1 \\
0.2713\end{array}$ & & $=-4$ & & $\begin{array}{r}\text { A.U. } \\
0.0000 \text { Toter }\end{array}$ & $\begin{array}{r}12 \text { cycl } \\
0.378\end{array}$ \\
\hline
\end{tabular}


Table s19. E-AMIDE calculated with DFT/AUG-cc-pVDZ

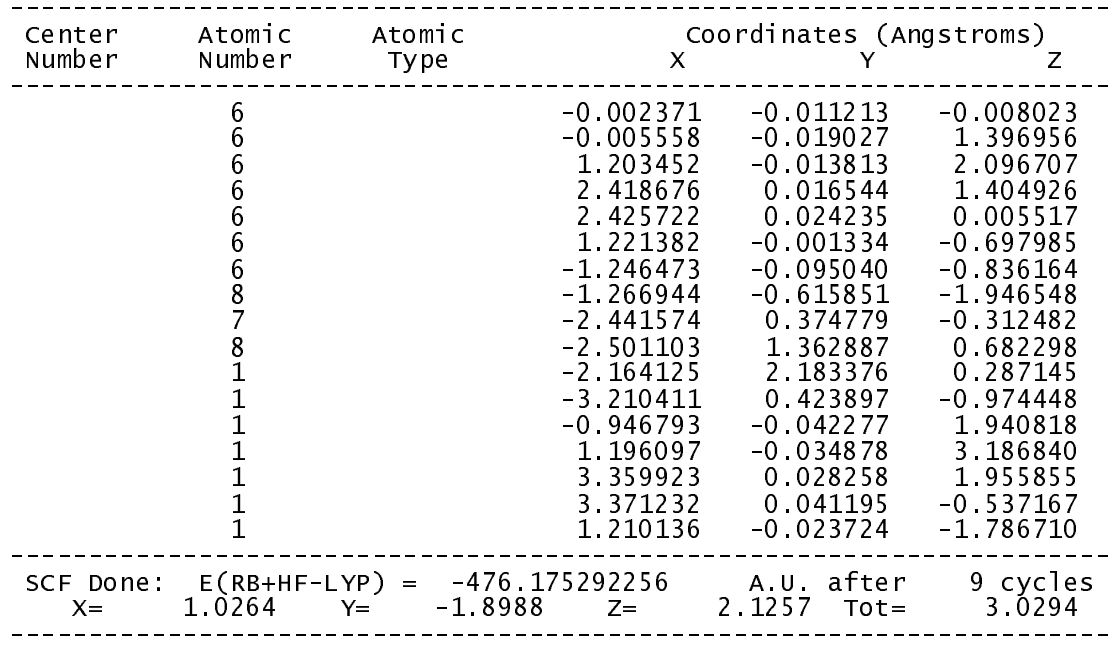

Table s20. E-IMIDE calculated with DFT/AUG-cc-pVDZ

\begin{tabular}{|c|c|c|c|c|c|}
\hline \multirow{2}{*}{$\begin{array}{l}\text { Center } \\
\text { Number }\end{array}$} & \multirow{2}{*}{$\begin{array}{l}\text { Atomic } \\
\text { Number }\end{array}$} & \multirow{2}{*}{$\begin{array}{l}\text { Atomic } \\
\text { Type }\end{array}$} & \multicolumn{3}{|c|}{ coordinates (Angstroms) } \\
\hline & & & $\mathrm{x}$ & r & Z \\
\hline & $\begin{array}{l}6 \\
6 \\
6 \\
6 \\
6 \\
6 \\
6 \\
8 \\
7 \\
8 \\
1 \\
1 \\
1 \\
1 \\
1 \\
1 \\
1\end{array}$ & & $\begin{array}{r}0.002020 \\
-0.001589 \\
1.203272 \\
2.426460 \\
2.440157 \\
1.242512 \\
-1.234138 \\
-0.991909 \\
-2.487075 \\
-2.774079 \\
-3.738751 \\
-1.857757 \\
-0.939591 \\
1.181848 \\
3.363054 \\
3.387973 \\
1.261262\end{array}$ & $\begin{array}{l}0.000000 \\
0.000000 \\
0.000000 \\
0.000000 \\
0.000000 \\
0.000000 \\
0.000000 \\
0.000000 \\
0.000000 \\
0.000000 \\
0.000000 \\
0.000000 \\
0.000000 \\
0.000000 \\
0.000000 \\
0.000000 \\
0.000000\end{array}$ & $\begin{array}{r}-0.010275 \\
1.399549 \\
2.105679 \\
1.430122 \\
0.031832 \\
-0.682050 \\
-0.823909 \\
-2.172514 \\
-0.522061 \\
0.855082 \\
0.857226 \\
-2.608490 \\
1.941241 \\
3.195845 \\
1.988868 \\
-0.507233 \\
-1.768572\end{array}$ \\
\hline $\begin{array}{l}\mathrm{CF} D \mathrm{DC} \\
X=\end{array}$ & $\begin{array}{l}\mathrm{E}(\mathrm{RB}+ \\
.1141\end{array}$ & $=$ & $\begin{array}{c}0479588 \\
\mathrm{Z}=\end{array}$ & $\begin{array}{l}\text { A.U. } \\
0.3847 \text { Tot }\end{array}$ & $\begin{array}{c}12 \text { cycles } \\
0.4013\end{array}$ \\
\hline
\end{tabular}

Table s21. Anion-Ia calculated with DFT/AUG-cc-pVDZ

\begin{tabular}{|c|c|c|c|c|c|c|c|c|}
\hline$C D$ & Cent & Atom N1 & & $\begin{array}{l}\text { Z-MATRI } \\
\text { eng th/X }\end{array}$ & (A & $\begin{array}{l}\text { NGSTROMS } \\
2 \quad \mathrm{~A} 7 \mathrm{pl}\end{array}$ & $\begin{array}{cc}\text { AND } & \text { DEGREES) } \\
\mathrm{ha} / \mathrm{Y} & \mathrm{N} 3\end{array}$ & Beta/z \\
\hline $\begin{array}{l}\mathrm{C} \\
\mathrm{C} \\
\mathrm{C} \\
\mathrm{C} \\
\mathrm{C} \\
\mathrm{C} \\
\mathrm{C} \\
\mathrm{O} \\
\mathrm{N} \\
\mathrm{O} \\
\mathrm{H} \\
\mathrm{H} \\
\mathrm{H} \\
\mathrm{H} \\
\mathrm{H} \\
\mathrm{H}\end{array}$ & $\begin{array}{r}1 \\
2 \\
3 \\
4 \\
5 \\
1 \\
7 \\
7 \\
9 \\
10 \\
2 \\
3 \\
4 \\
5 \\
6\end{array}$ & $\begin{array}{l}1.409305 \\
1.395746 \\
1.404079 \\
1.398885 \\
1.399846 \\
1.500717 \\
1.283356 \\
1.325957 \\
1.424736 \\
0.988526 \\
1.089051 \\
1.092872 \\
1.092242 \\
1.092991 \\
1.089674\end{array}$ & $\begin{array}{l}1 \\
2 \\
3 \\
4 \\
2 \\
1 \\
1 \\
7 \\
9 \\
1 \\
2 \\
3 \\
4 \\
5\end{array}$ & $\begin{array}{l}120.824 \\
120.529 \\
119.072 \\
120.387 \\
122.879 \\
120.017 \\
114.974 \\
108.610 \\
101.430 \\
118.260 \\
119.677 \\
120.420 \\
119.862 \\
121.581\end{array}$ & $\begin{array}{l}1 \\
2 \\
3 \\
3 \\
2 \\
8 \\
8 \\
7 \\
6 \\
1 \\
2 \\
3 \\
4\end{array}$ & $\begin{array}{r}0.000 \\
0.000 \\
0.000 \\
180.000 \\
180.000 \\
180.000 \\
0.000 \\
0.000 \\
180.000 \\
180.000 \\
180.000 \\
180.000 \\
180.000\end{array}$ & $\begin{array}{l}0 \\
0 \\
0 \\
0 \\
0 \\
0 \\
0 \\
0 \\
0 \\
0 \\
0 \\
0 \\
0\end{array}$ & \\
\hline SCF & $\begin{array}{l}\text { Done } \\
\text { ole } \mathrm{m} \\
\mathrm{X}=\end{array}$ & $\begin{array}{r}\mathrm{E}(\mathrm{RB}+\mathrm{H} \\
\text { nent (Deb } \\
7.4162\end{array}$ & $Y=$ & $\begin{array}{r}=-475 \\
\quad 0.00\end{array}$ & 1 & $\mathrm{Z}=$ & $\begin{array}{l}\text { A.U. after } \\
3.3948 \text { Tot }=\end{array}$ & $\begin{array}{r}10 \text { cycles } \\
8.1563\end{array}$ \\
\hline
\end{tabular}


Table s22. Anion IIa calculated with DFT/AUG-cc-pVDZ

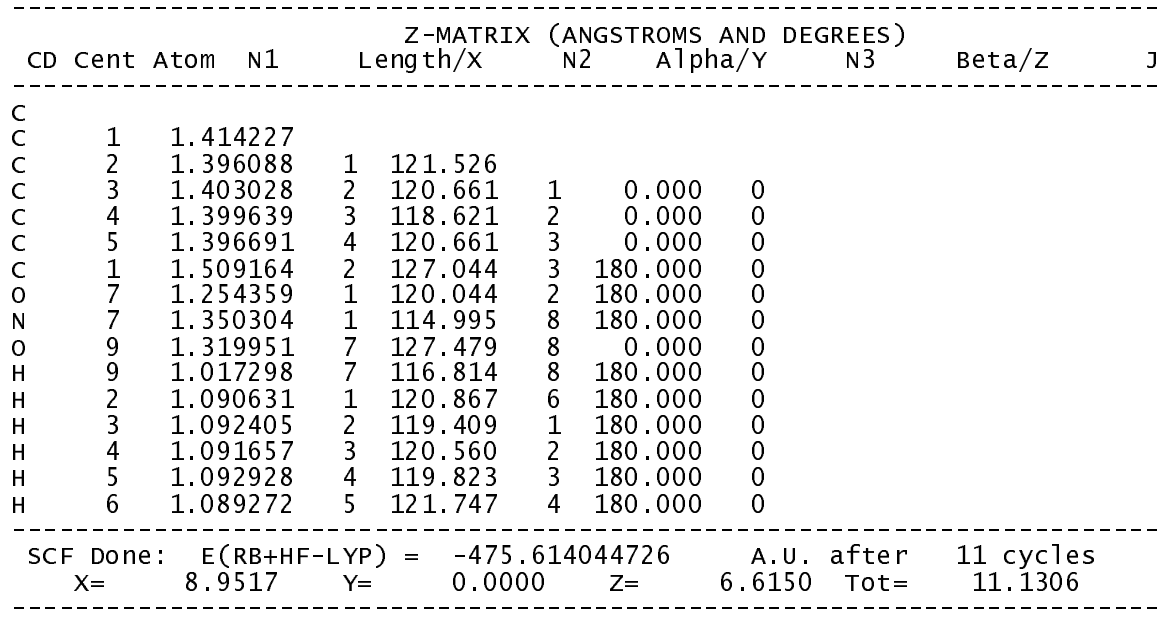

Table s23. Anion Ib calculated with DFT/AUG-cc-pVDZ

\begin{tabular}{|c|c|c|c|c|c|c|c|c|}
\hline$C D$ & cent & Atom $\quad \mathrm{N} 1$ & & $\begin{array}{l}\text { Z-MATRIX } \\
\text { ength/X }\end{array}$ & $(A$ & $\begin{array}{l}\text { NGSTROMS } \\
2 \quad \text { Al } 1 \mathrm{pr}\end{array}$ & $\begin{array}{cc}\text { AND } & \text { DEGREES) } \\
\mathrm{ha} / \mathrm{Y} & \mathrm{N} 3\end{array}$ & Beta/z \\
\hline $\begin{array}{l}\mathrm{C} \\
\mathrm{C} \\
\mathrm{C} \\
\mathrm{C} \\
\mathrm{C} \\
\mathrm{C} \\
\mathrm{C} \\
\mathrm{O} \\
\mathrm{N} \\
\mathrm{O} \\
\mathrm{H} \\
\mathrm{H} \\
\mathrm{H} \\
\mathrm{H} \\
\mathrm{H} \\
\mathrm{H}\end{array}$ & $\begin{array}{l}1 \\
2 \\
3 \\
4 \\
5 \\
1 \\
7 \\
7 \\
9 \\
8 \\
2 \\
3 \\
4 \\
5 \\
6\end{array}$ & $\begin{array}{l}1.419476 \\
1.390437 \\
1.407767 \\
1.399625 \\
1.397759 \\
1.446728 \\
1.367903 \\
1.318311 \\
1.335065 \\
1.016433 \\
1.089150 \\
1.093014 \\
1.091559 \\
1.093152 \\
1.089054\end{array}$ & $\begin{array}{l}1 \\
2 \\
3 \\
4 \\
2 \\
1 \\
1 \\
7 \\
7 \\
1 \\
2 \\
3 \\
4 \\
5\end{array}$ & $\begin{array}{r}120.997 \\
121.015 \\
118.548 \\
120.912 \\
121.655 \\
119.326 \\
123.387 \\
111.213 \\
96.011 \\
118.139 \\
119.303 \\
120.685 \\
119.780 \\
120.438\end{array}$ & $\begin{array}{l}1 \\
2 \\
3 \\
3 \\
2 \\
8 \\
8 \\
9 \\
6 \\
1 \\
2 \\
3 \\
4\end{array}$ & $\begin{array}{r}0.000 \\
0.000 \\
0.000 \\
180.000 \\
180.000 \\
180.000 \\
0.000 \\
0.000 \\
180.000 \\
180.000 \\
180.000 \\
180.000 \\
180.000\end{array}$ & $\begin{array}{l}0 \\
0 \\
0 \\
0 \\
0 \\
0 \\
0 \\
0 \\
0 \\
0 \\
0 \\
0 \\
0\end{array}$ & \\
\hline & $\begin{array}{l}\text { F Do } \\
\text { pole } \\
X=\end{array}$ & $\begin{array}{c}E(\mathrm{RB}+\mathrm{H} \\
\text { ment }(\mathrm{De} \\
8.0353\end{array}$ & $\mathrm{Y}=$ & $\begin{array}{r}=-475 . \\
0.000\end{array}$ & 0 & $\begin{array}{l}49013 \\
z=\end{array}$ & $\begin{array}{r}\text { A.U. } \text { after } \\
2.6035 \text { Tot }=\end{array}$ & $\begin{array}{r}11 \text { cycle } \\
8.4466\end{array}$ \\
\hline
\end{tabular}

Table s24. ANION IIb DFT minimum

\begin{tabular}{|c|c|c|c|c|c|c|c|c|}
\hline$C D$ & cent & Atom $\mathrm{N} 1$ & & $\begin{array}{l}\text { Z-MATRIX } \\
\text { ength } / x\end{array}$ & (A & $\begin{array}{l}\text { NGSTROMS } \\
2 \quad \mathrm{~A} 7 \mathrm{p}\end{array}$ & $\begin{array}{cc}\text { AND } & \text { DEGREES) } \\
\mathrm{ha} / \mathrm{Y} & \mathrm{N} 3\end{array}$ & Beta/Z \\
\hline $\begin{array}{l}\mathrm{C} \\
\mathrm{C} \\
\mathrm{C} \\
\mathrm{C} \\
\mathrm{C} \\
\mathrm{C} \\
\mathrm{O} \\
\mathrm{N} \\
\mathrm{O} \\
\mathrm{H} \\
\mathrm{H} \\
\mathrm{H} \\
\mathrm{H} \\
\mathrm{H} \\
\mathrm{H}\end{array}$ & $\begin{array}{l}1 \\
2 \\
3 \\
4 \\
5 \\
1 \\
7 \\
7 \\
9 \\
9 \\
2 \\
3 \\
4 \\
5 \\
6\end{array}$ & $\begin{array}{l}1.411372 \\
1.402186 \\
1.399866 \\
1.402834 \\
1.397450 \\
1.515976 \\
1.264612 \\
1.341829 \\
1.349669 \\
1.021514 \\
1.089310 \\
1.093211 \\
1.093277 \\
1.093038 \\
1.089624\end{array}$ & $\begin{array}{l}1 \\
2 \\
3 \\
4 \\
2 \\
1 \\
1 \\
7 \\
7 \\
1 \\
2 \\
3 \\
4 \\
5\end{array}$ & $\begin{array}{l}120.256 \\
120.859 \\
119.213 \\
120.160 \\
125.266 \\
119.440 \\
119.850 \\
131.922 \\
112.439 \\
117.269 \\
119.374 \\
120.423 \\
119.998 \\
121.416\end{array}$ & $\begin{array}{l}1 \\
2 \\
3 \\
3 \\
2 \\
8 \\
8 \\
8 \\
6 \\
1 \\
2 \\
3 \\
4\end{array}$ & $\begin{array}{r}0.000 \\
0.000 \\
0.000 \\
180.000 \\
180.000 \\
180.000 \\
180.000 \\
0.000 \\
180.000 \\
180.000 \\
180.000 \\
180.000 \\
180.000\end{array}$ & $\begin{array}{l}0 \\
0 \\
0 \\
0 \\
0 \\
0 \\
0 \\
0 \\
0 \\
0 \\
0 \\
0 \\
0\end{array}$ & \\
\hline SC & $\begin{array}{l}\text { Don } \\
\text { ole } \\
X=\end{array}$ & $\begin{array}{c}\mathrm{E}(\mathrm{RB}+\mathrm{HF} \\
\text { nent (Deb) } \\
6.4378\end{array}$ & $\mathrm{Y}=$ & $\begin{array}{r}=-475.6 \\
0.000\end{array}$ & 0 & $\begin{array}{c}5256 \\
Z=\end{array}$ & $\begin{array}{l}\text { A.U. after } \\
0.9128 \text { Tot }=\end{array}$ & $\begin{array}{r}22 \text { cycles } \\
6.5022\end{array}$ \\
\hline
\end{tabular}


Table S25. E-anionIa calculated with DFT/AUG-cc-pVDZ

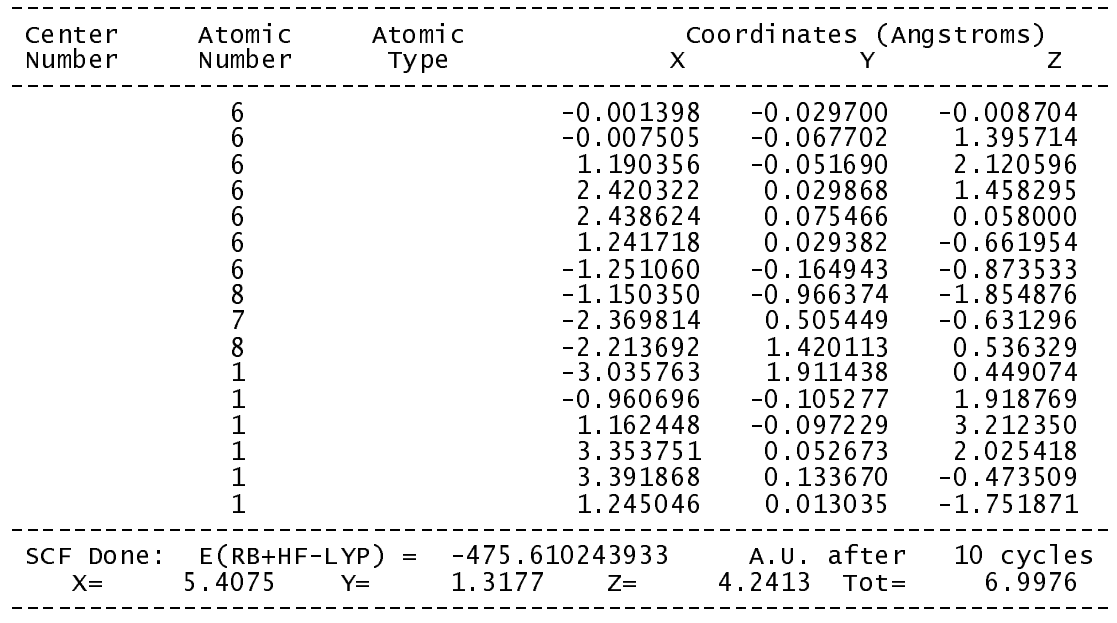

Table s26. E-anionIb calculated with DFT/AUG-cc-pVDZ

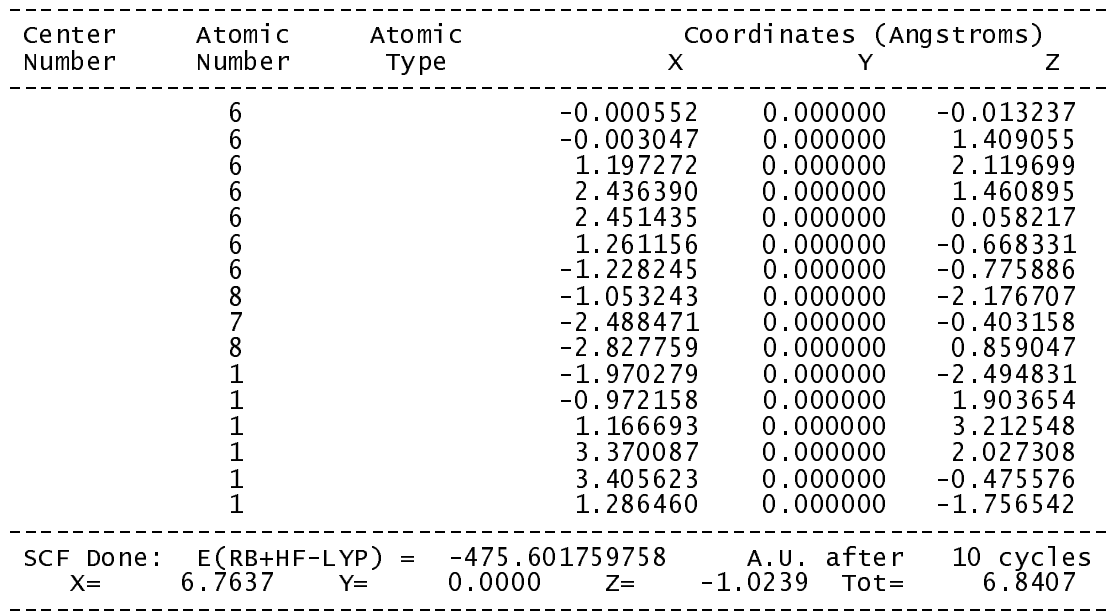

Table s27. E-anionII calculated with DFT/AUG-cc-pVDZ

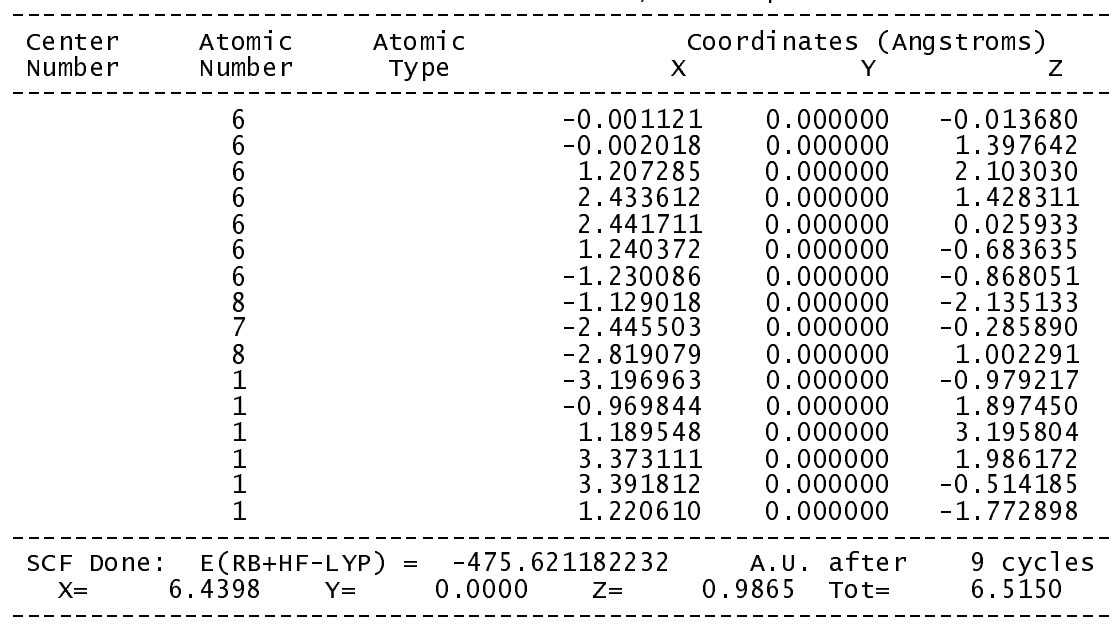


Figure s1. ${ }^{1}{ }^{-1}{ }^{1}$ HNMR $2 \mathrm{D}$ NOESY of BHA in acetone $d_{6} . T=-60^{\circ} \mathrm{C}, \mathrm{C}_{\mathrm{BHA}}=0.032 \mathrm{M}$

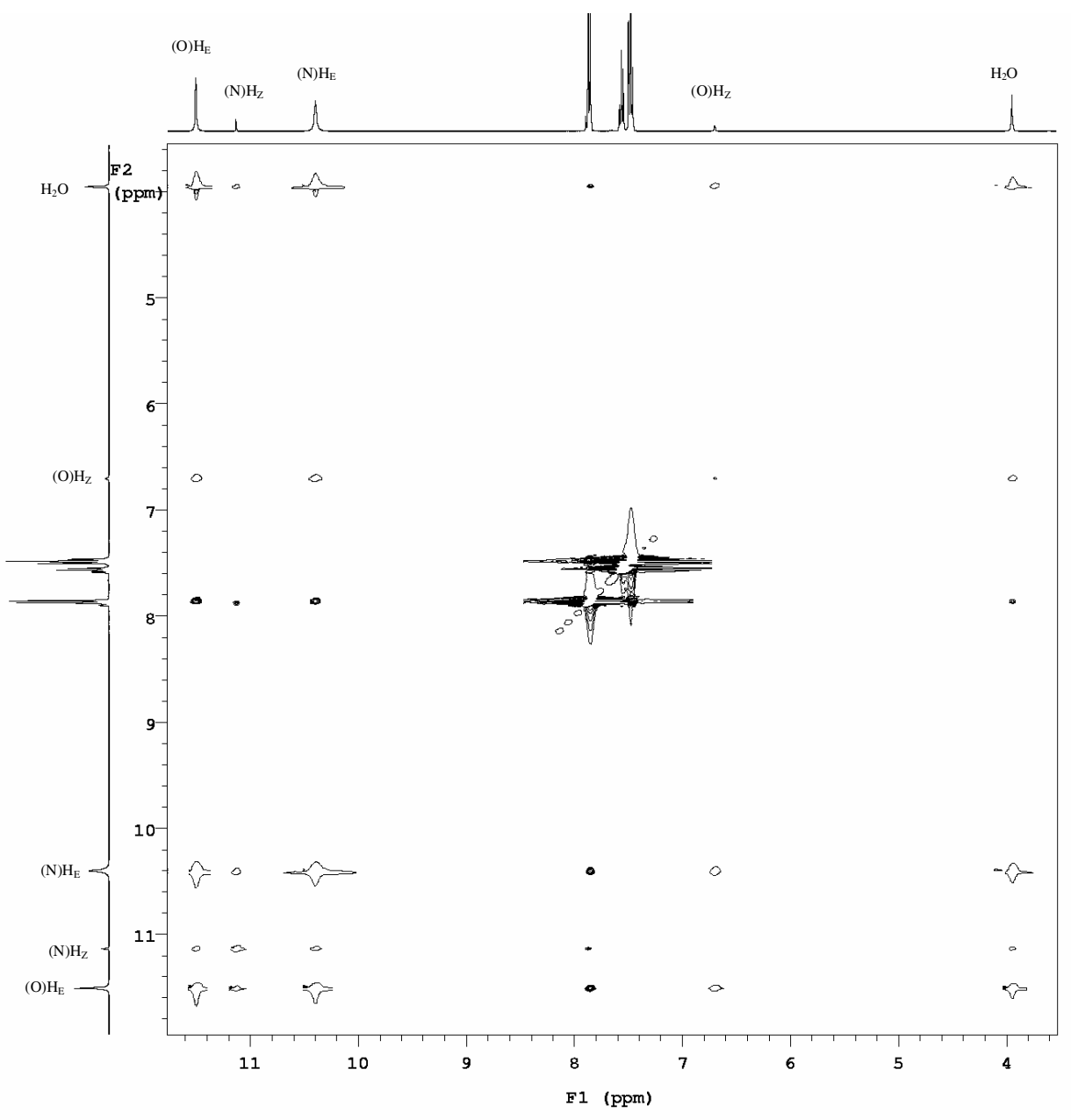


Figure s2. ${ }^{1} \mathrm{HNMR}$ spectra of $\mathrm{BHA}$ in acetone $\mathrm{d}_{6}$ between $-60^{\circ} \mathrm{C}$ and $20^{\circ} \mathrm{C} . \mathrm{C}_{\mathrm{BHA}}=0.032 \mathrm{M}$
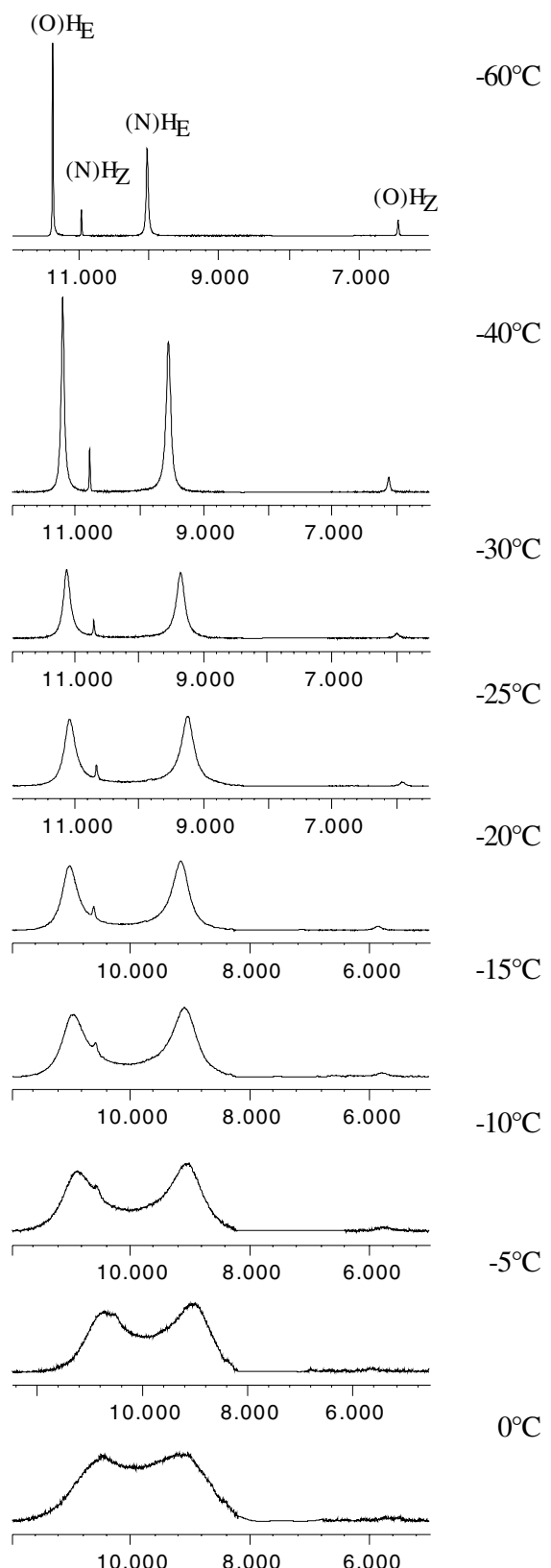

$0^{\circ} \mathrm{C}$
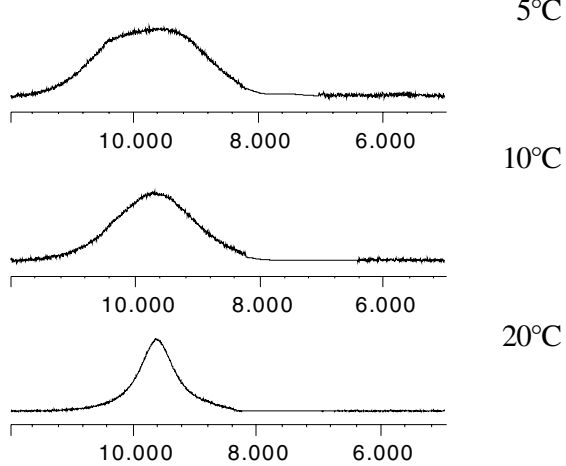
Figure s3. Stopped-flow kinetic curve for the Ni(II)/BHA system. $C_{L}=4 \times 10^{-5} \quad M, C_{M}=$ $6 \times 10^{-3} \mathrm{M},\left[\mathrm{H}^{+}\right]=1.2 \times 10^{-6} \mathrm{M}, I=0.2 \mathrm{M}\left(\mathrm{NaCl0}_{4}\right), \mathrm{T}=25^{\circ} \mathrm{C}, \lambda=280 \mathrm{~nm}$, pretrigger $=0.05$ s.

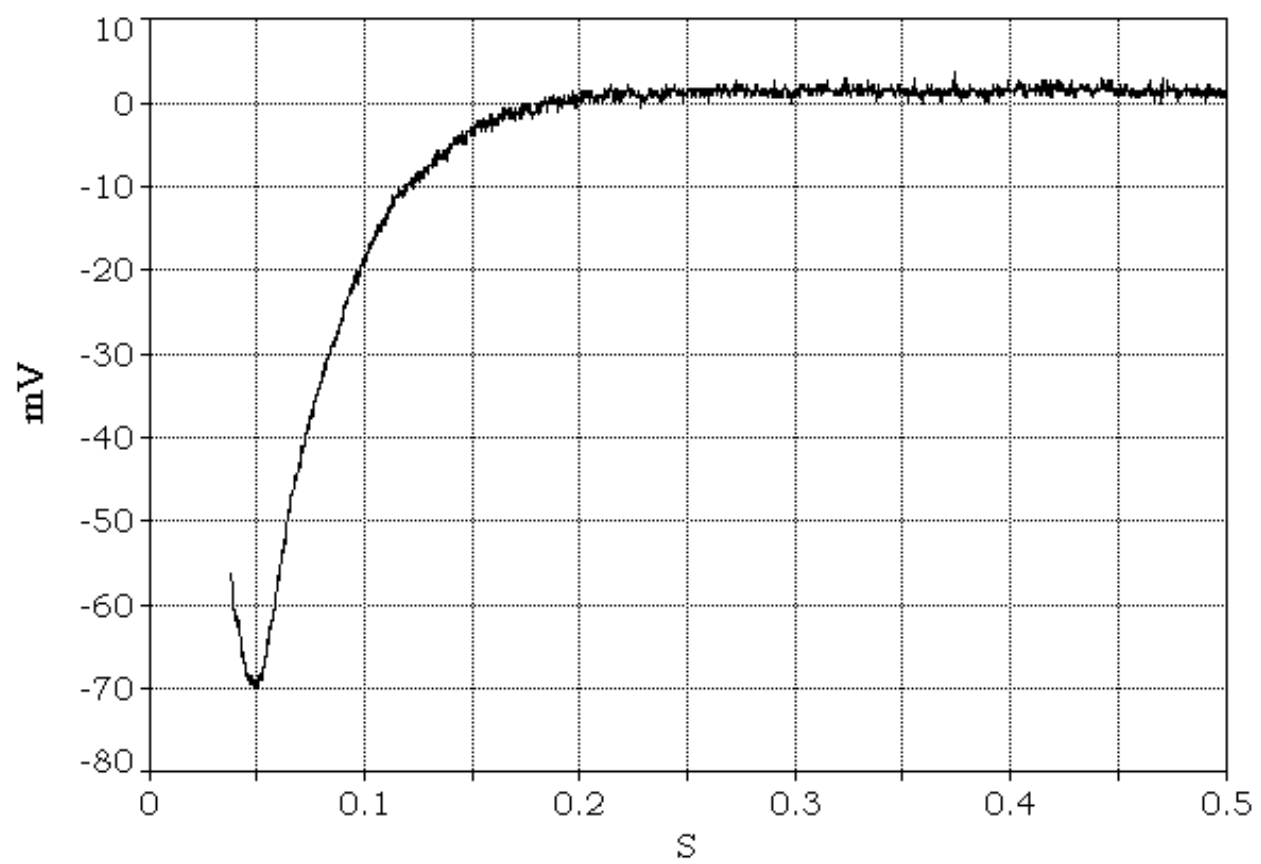

